\title{
FASTEN: an loT platform for manufacturing. Embraer use case
}

\author{
Ricardo Reis ${ }^{1, *}$, Flávio Diniz ${ }^{2}$, Luciana Mizioka ${ }^{2}$, Paula Olivio $^{2}$, Gléverson Lemos ${ }^{2}$, Marta \\ Quintiães $^{1}$, Ruben Menezes ${ }^{1}$, Flávio Amadio $^{2}$, Narciso Caldas ${ }^{3}$ \\ ${ }^{1}$ Embraer Engineering and Technology Center, Embraer Portugal SA, PIAE Lote AI, Herdade do \\ Pinheiro e Casa Branca, 7005-797 Évora, Portugal \\ ${ }^{2}$ Embraer SA, Avenida Brigadeiro Faria Lima, 2170, CEP 12227-901, São José dos Campos, Brasil \\ ${ }^{3}$ INES-TEC, R. Dr. Roberto Frias, 4200-465 Porto, Portugal
}

\begin{abstract}
FASTEN is an $\mathrm{H} 2020$ project under a bilateral call UE-Brazil. It aims to advance IoT and IoT enabled applications to support Industry 4.0 concepts, namely in the area of automation and additive manufacturing. The project results will be demonstrated through two pilots: one in Brazil, lead by a ThyssenKrupp use case, and the other in Europe, at Embraer facilities in Portugal. The detail on the Embraer use case, on pick'n'place automation, predictive and prescriptive analytics and assembly line simulation are described as FASTEN architecture and approach.
\end{abstract}

\section{Introduction}

The lowering of costs of processing power, memory, communication and sensors is opening the perspective of transforming the collection of ever-increasing pools of data into actionable information and knowledge. This happens horizontally and vertically, not only with huge data pools but also in real-time, like a living cyber-physical system, connecting the full value chain across the product life. Concepts as "smart factories", "industry 4.0", "digital thread" have become the industry buzzwords in the last years.

The need to bring innovative products to market faster than ever is transversal to all industries. In Aerospace, with its long development times, high capital investment and long life cycles, the benefits of digital integration of cyber-physical systems have long been understood and sought. Demands for limiting quality costs and cycle times, excluding human factors as a source of error as far as possible, and become more efficient, are key drivers to achieve that goal. Continuous recording and analysis of manufacturing process data allow systems failures to be predicted and inefficient developments to be corrected in order to improve productivity (reducing lead times). These smart factory and smart supply concepts also connect with ongoing efforts to integrate with product development and design. As such, not only higher efficiencies for manufacturing are sought but, ultimately, a more sustainable world and usher the implementation of product changes and upgrades, bringing to society the benefits of new and improved solutions in traditionally conservative industries.

\footnotetext{
* Corresponding author: rireis@pt.embraer.com
} 
The vision of Industry 4.0 and similar concepts is enticing and, although always aware of the hype, it is achievable. Nevertheless, a long road lays ahead until it will fully come to fruition. The issues of integration of new and legacy systems, automation, cybersecurity, algorithms for data processing, and human-machine interfaces for effective support to the human decision are only just a few that must be dealt with. Underlying all is the use of common reference architectures, true foundations upon which to set the lay the building bricks.

FASTEN ultimate goal is to contribute to such kind of foundations. Namely, it is an H2020 project funded under a bilateral call UE-Brazil [1], to promote common frameworks and standards for Industrial Internet of Things (IIoT) based platforms for manufacturing between the two regions. It will thus develop and integrate a host of technologies to demonstrate concrete results for the Industry 4.0 paradigm.

This paper describes the FASTEN project and one of its use cases, lead by Embraer. This use case will be demonstrated at an Embraer facility in Portugal, a literal example of collaboration between Europe and Brazil.

\section{FASTEN}

\subsection{Ambition and goals}

FASTEN - Flexible and Autonomous Manufacturing Systems for Custom-Designed Products - is an H2020 project under the bilateral call UE-Brazil for IoT pilot lines for customization and smart Manufacturing (H2020-EUB-2017). Its strategic goals are:

- Foster digital manufacturing sustainability and be an enabler of technologies development between Brazil and Europe;

- Provide a multi-disciplinary decision support tool to improve trade-off analysis;

- $\quad$ Contribute to the competitiveness of Brazil and Europe.

To achieve these goals a set of specific objectives were defined, framed by two industrial use cases relevant to their sponsors: ThyssenKrupp and Embraer. These goals are:

- Develop and demonstrate a flexible and scalable robotic system and its integration with mass customization production lines;

- Standardize data repository and decision-making integration, from end-users (consumers) services to the manufacturing and supply levels;

- Optimize, synchronize and improve the coordination in real-time of the production and logistics activities;

- Validate and demonstrate the FASTEN Framework in two cross-sectorial industrial pilot cases;

- Improve the overall supply-chain performance and decision-making effectiveness;

- Validate technology and IoT architectures.

The final result of these specific objectives will be the instantiation of the FASTEN reference architecture in two pilot demonstrators, one in Europe, the other in Brazil.

\subsection{Consortium}

FASTEN is pursued by a consortium of partners distributed through Europe and Brazil. INESC-TEC coordinates the group of European partners, and INESC-P\&D those in Brazil. ThyssenKrupp leads the use case in Brazil, Embraer Portugal is responsible for the European use case. The remaining consortium partners are shown in Fig. 1. 


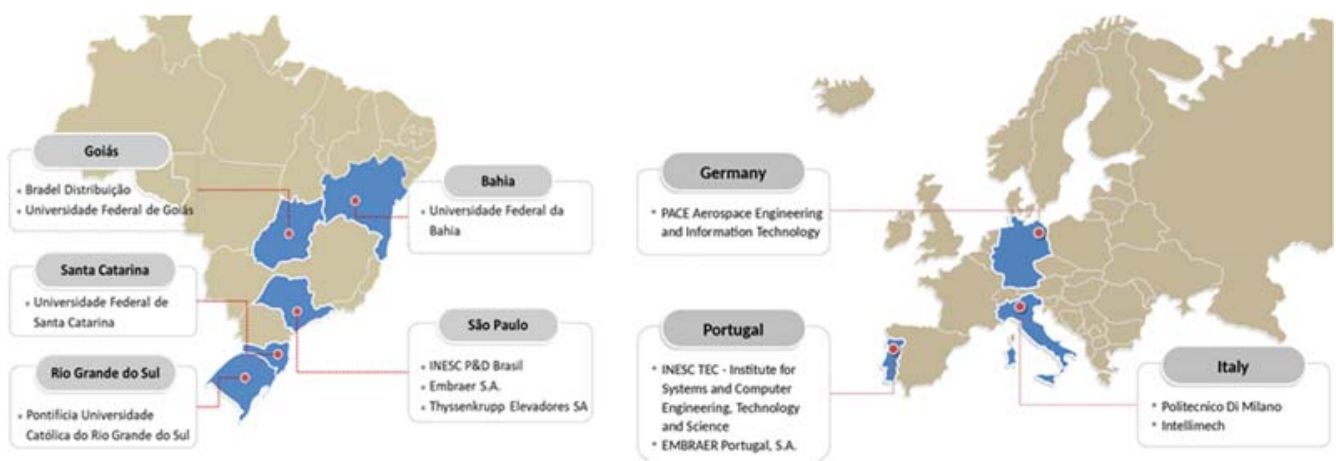

Fig. 1 FASTEN partners in Europe and Brazil.

\subsection{Methodology}

FASTEN is structured on top of two use cases, one in the area of additive manufacturing (WP5), the other in robotics (WP6). These use cases drive and frame the core components of FASTEN, namely: WP2) the robot and additive manufacturing systems integration, WP3) the open industrial IoT platform; and WP4) the predictive real-time simulation and optimization (Fig. 2.).

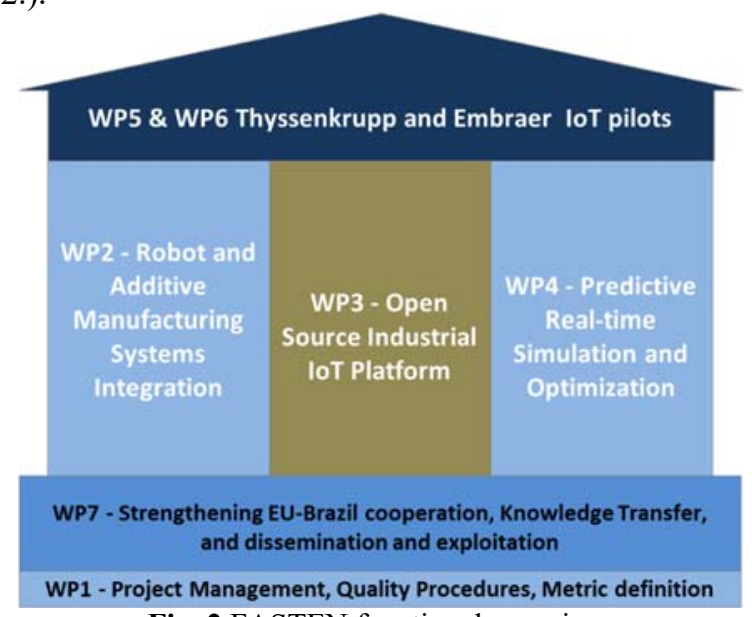

Fig. 2 FASTEN functional overview.

The tools in WO4 are the FASTEN Industrial Analytics Suite, seen in Fig. 3.

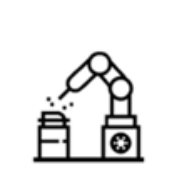

Flexible and scalable robotic and additive manufacturing system WP2

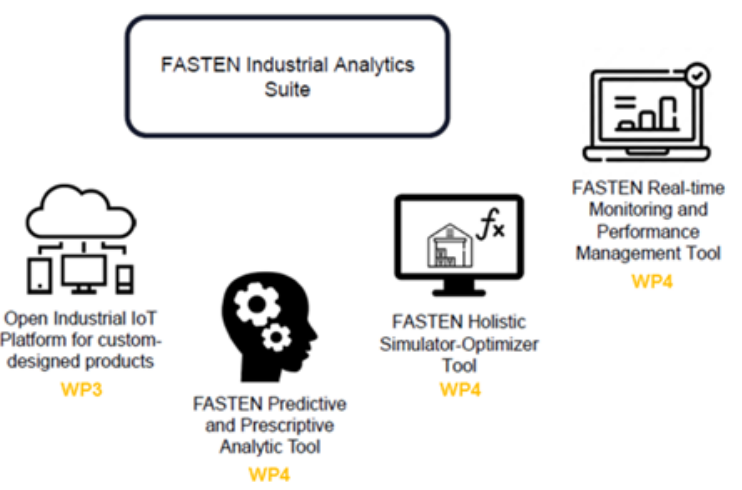

Fig. 3 FASTEN Industrial Analytics Suite 


\subsection{FASTEN architecture: RAMI 4.0}

A core objective of FASTEN is to propose an open RAMI 4.0 architecture [2]. The RAMI 4.0 - Reference Architectural Mode for Industrie 4.0 - was developed in Europe to enable the Industry 4.0 concept. It describes a three-dimensional model, organizing the lifecycle/value streams and the manufacturing hierarchy levels across the six layers of the IT representation of Industry 4.0. One of the main objectives is to facilitate the communication of the scope and design of the system. This will support collaboration and integration with other relevant initiatives by framing the developed concepts and technologies in a common model.



Fig. 4 RAMI 4.0 architecture (from [1])

Similar initiatives exist in the USA - the Industrial Internet Reference Architecture (IIRA) - and Japan - the Industrial Value Chain Reference Architecture. The convergence of RAMI 4.0 and IIRA was considered the most promising avenue to exploit within FASTEN.

\section{Embraer use case}

\subsection{Embraer in FASTEN}

Embraer is the third largest aircraft Original Equipment Manufacturer (OEM). It operates in a highly competitive environment, designing, producing and certifying products of great complexity and value, with long life cycles $\dagger$. As such, Embraer has awareness of the possibilities brought by digitalization, either for improving its processes or to create a digital environment able to better tie up operation, manufacturing, and design.

Besides contributing to long-term goals, being able to deliver medium/short term gains to the production environment is a much-desired synergy in a development process. The FASTEN Embraer Use Case aims to exercise the proposed solutions in an environment representative of real operation, similar to fast prototyping, evaluate their effectiveness to deliver on expected key performance indicators.

The Embraer Use Case can thus be framed by a set of high level driving questions:

1. How to design, build and deploy an Industrial IoT architecture that cross-links physical with digital assets?

2. How to integrate into this architecture legacy and new systems?

\footnotetext{
$\dagger$ Aircraft can span more than 30 years in service.
} 
3. How to leverage real-time (in motion) and permanent data (at rest) for management, process and design improvement?

4. How to incorporate and use open architecture and open-source approaches into a controlled industrial environment?

Embraer high-level goals for FASTEN can be expressed as follows:

1. Obtain an adaptive, smart, extendable robotic solution for picking and handling of generic parts;

2. Obtain models for predictive and prescriptive algorithms to support Prognostics Health Management (PHM) and Condition Based Maintenance (CBM) paradigms for maintenance and understand how to integrate them within an IoT architecture;

3. Understand how to couple different systems and data streams - real and synthetic, real-time and permanent - with simulation and optimization for decision making support;

4. Explore industrial cockpit concepts to show different levels of information to different decision makers and stakeholders.

To evaluate FASTEN solutions to these driving questions, three scenarios were designed and the facilities of Embraer in Évora, Portugal, selected for a pilot demonstration. These are Centres of Excellence for Composite and Metallic aero-structures, specialized in wings and empennages manufacturing. Their main characteristics are the production of large, critical and/or complex parts (the biggest inside the Embraer group) with a high degree of automation and digital integration.
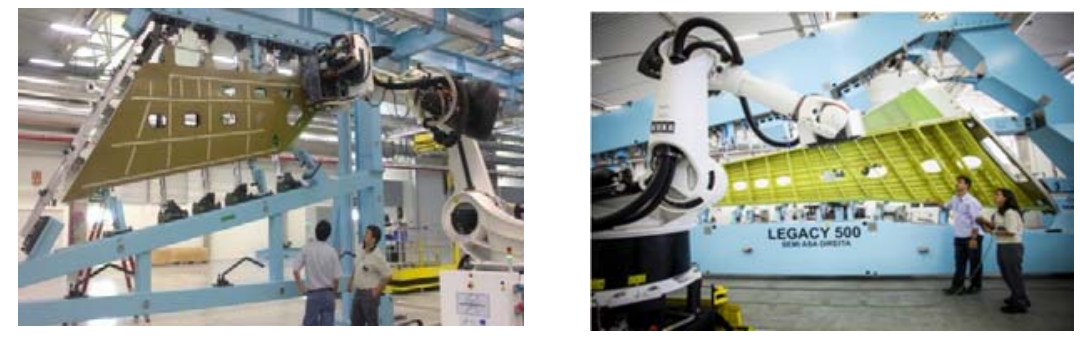

Fig. 5 Example of empennage (left) and wing (right) assembly at Évora using drilling and fastening robots.

\subsection{FASTEN Embraer use case: a global overview}

To better provide an understanding of the whole of FASTEN, an overarching scenario was designed, based on the operations at Embraer facilities. This provides a birds-eye view that facilitates understanding and integration of the different components, as depicted in Fig. 6. This showcases the flow of components from the automated warehouse, where a robotic solution for pick'n'place will be developed. The kits thus assembled will feed the assembly line. All robots will provide data through the IoT layer to be processed by the Predictive algorithms that incorporate the Robotic Health Monitoring Systems (RHMS). To manage forecasted disruption, an Assembly Line Simulator can be used to forecast the line behavior or to adapt it to improved or new products. 

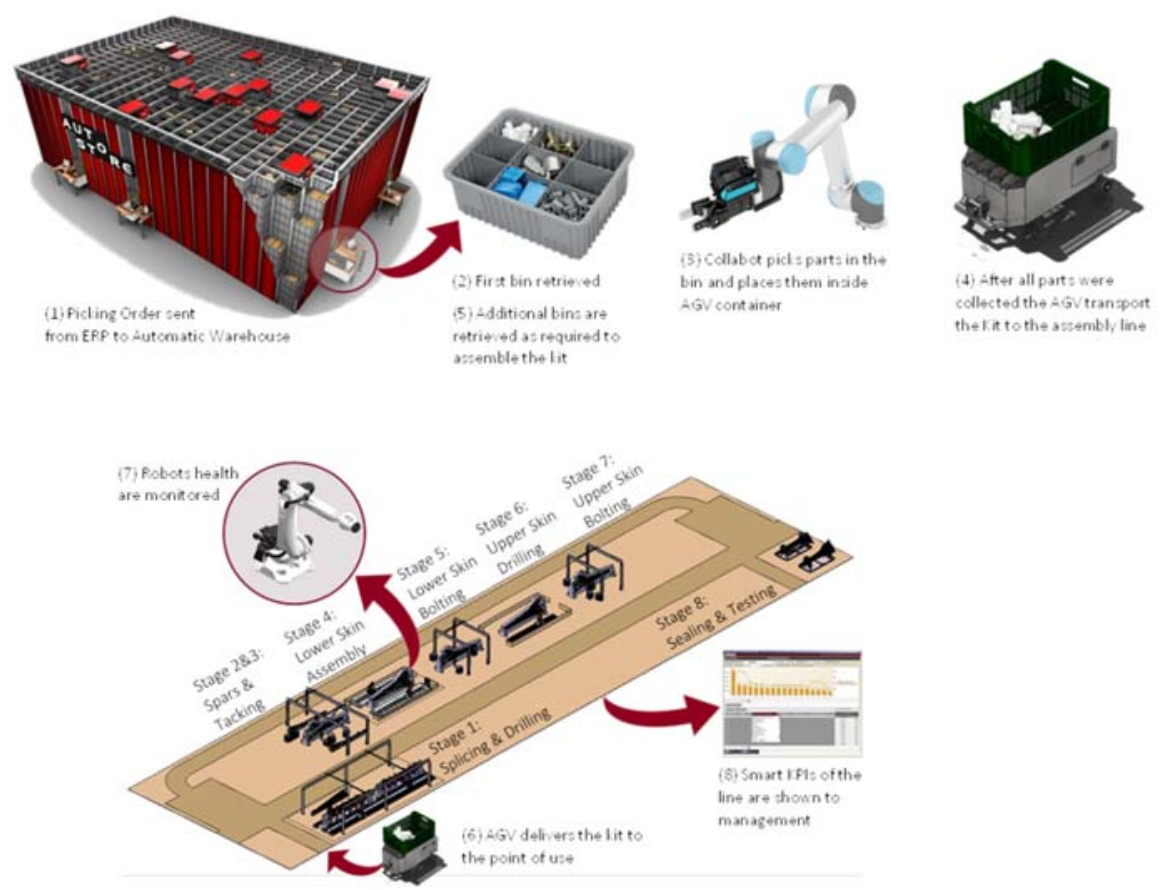

Fig. 6 The concept scenario for Embraer FASTEN use case.

\subsection{Adaptive pick and place robot for logistics}

The largest number of parts in an aero-structure assembly is of small size, with a huge spectrum of shapes, sizes, weights, and packagings. Embraer has several solutions of automated warehouses and uses a supply strategy to the assembly line based on assembly kits. These kits are set up by workers and placed in cars for expedition to the assembly line.

The ability to automate kit assembly using robots would free human workers for higher value tasks and increase process quality. This has been proven challenging for robotics due to the different characteristics of the parts as mentioned. The challenge of FASTEN is to deliver a smart solution, allowing the robot to pick a generic part and quickly learn to pick new parts. This solution will be integrated using the IoT architecture developed by FASTEN for seamless operation with human workers and other digital systems in the shop floor.

\subsection{Wing assembly line simulation and optimization}

A simulator for the wing assembly line is sought. This simulator will support disruption management or re-configuration of the line due to the introduction of new products.

The use of Simulation and Optimization tools allows the user to optimize, simulate and analyze the behavior of a production system, helping with decision-making tasks. For the Embraer wing assembly line, this type of tool will allow the user to test different scenarios and get optimized results and decisions according to input parameters, such as workstation processing times, different operation routes and transportation times. The synchronization with the current status of the manufacturing line (work-in-process), achieved by receiving 
data through the IoT Platform, will allow the optimization and simulation activities to be accurate. Also, the ability to use historical data to improve the simulator will be developed.

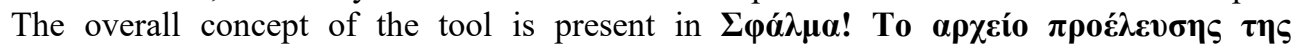

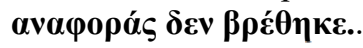

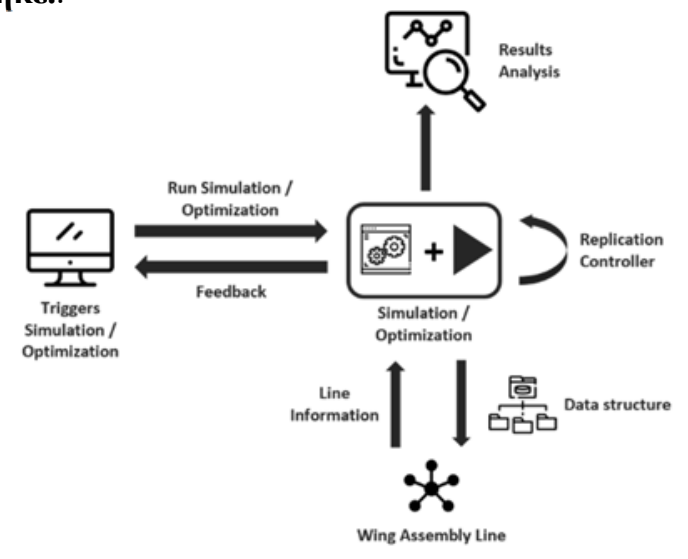

Fig. 7 Concept Holistic Simulation and Optimization Tool for Embraer FASTEN use case.

\subsection{Self- adaptive predictive analytics for robot health monitoring system}

At Embraer Portugal, part of the manufacturing process is automated by robots/machines to provide aircraft parts with high quality and repeatability, at a lower cost and faster compared to manual processes. This context frames the scenario, where a component designated Robot Health Management System (RHMS) will harness the data generated by the robots, through the IoT layer, to predict remaining the useful life (RUL) of their components. This ability is key to advanced maintenance concepts like Condition Based Maintenance (CBM).

The methodologies of Self-Adaptive Predictive and Prescriptive Analytics will be used to predict unknown future events through coupling of mathematical models with the data collected from the robots (predictive) and information on how to recover the normal operation (prescriptive). Additionally, for the RHMS, like for the previous scenario, the proposal is to develop a framework that allows the predictive models to improve themselves, based on historical robot data and of the processes associated with the robot operation.

\section{Results}

The FASTEN open reference architecture is already a result of the project. It specifies the different functional components and their interfaces. It is based on RAMI 4.0. It allows the selection and integration of 1) elements develop, 2) those other initiatives like FIWARE, ROS or Apache, and 3) proprietary corporate and industrial systems, always present in the operational setting. An overview of the FASTEN open architecture is given in Fig. 8. 


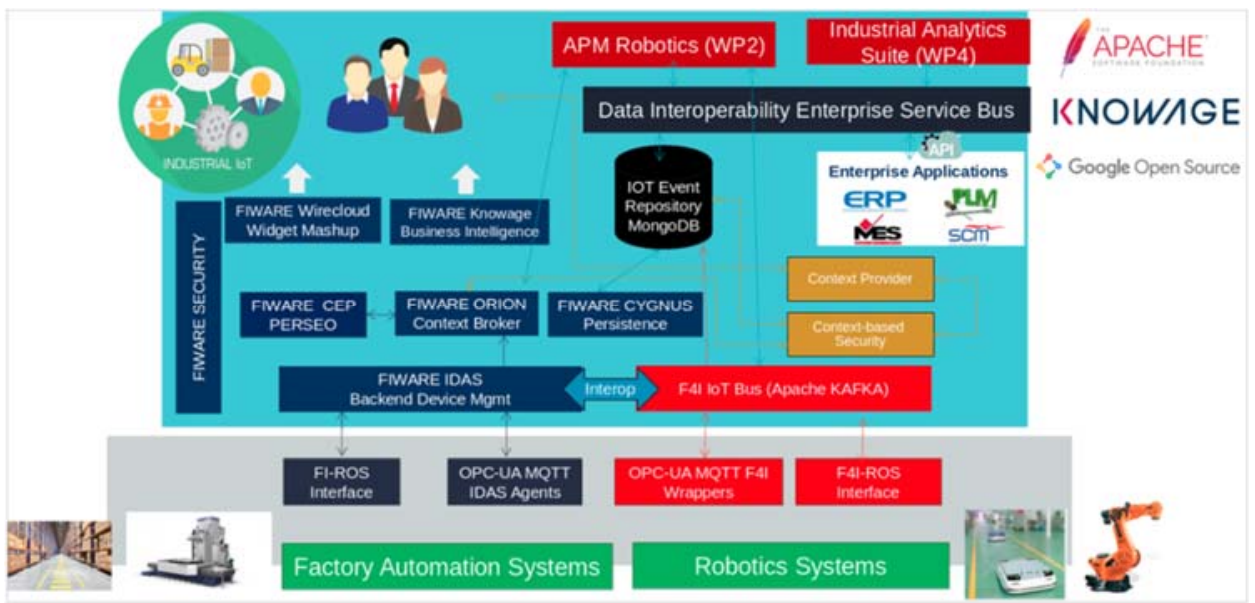

Fig. 8 FASTEN reference architecture

\section{Final comments}

Douglas Engelbart, once said "The key thing about all the world's big problems is that they have to be dealt with collectively. If we don't get collectively smarter, we're doomed". The manufacturing industry, which designs, develops and builds the complex systems that support society, face ever-increasing challenges in the fast-changing world within tighter constraints. This is more acutely felt in industries like aerospace, of high capital intensity, long developing times and life cycles.

FASTEN main purpose is thus to further the progress of digitalization, enabling manufacturing technologies like additive manufacturing and automation to be smartly integrated into a cyber-physical fabric. Their data and controls will be connected through IoT, transforming data into knowledge, and better support decision making for flexibility and efficiency. By design FASTEN does this through collaboration, using open reference architectures (RAMI 4.0), and exercising joint development with European and Brazilian industry and researchers. This collaborative, networked, use case driven approach will one expects - accelerate innovation and value creation to all.

\section{Acknowledgments}

This work is being partially supported by H2020, project grant 777096, FASTEN - Flexible and Autonomous Manufacturing Systems for Custom-Designed Products.

\section{References}

1. https://ec.europa.eu/research/participants/portal/desktop/en/opportunities/h2020/topics/ eub-02-2017.html (retrieved 2018.09.01)

2. Reference Architecture Model Industrie 4.0 (RAMI4.0), Status Report, VDI, 2015, https://www.zvei.org/fileadmin/user_upload/Themen/Industrie_4.0/Das_Referenzarchi tekturmodell_RAMI_4.0_und_die_Industrie_4.0-

Komponente/pdf/5305_Publikation_GMA_Status_Report_ZVEI_Reference_Architect ure_Model.pdf 